\title{
Main shocks and evolution of complex earthquake networks
}

\author{
Sumiyoshi Abe \\ Department of Physical Engineering, Mie University, Mie 514-8507, Japan \\ Institut Supérieur des Matériaux et Mécaniques Avancés, 44 F. A. Bartholdi, 72000 Le Mans, France \\ Norikazu Suzuki \\ College of Science and Technology, Nihon University, Chiba 274-8501, Japan
}

(Received on 5 March, 2009)

\begin{abstract}
Dynamical evolution of earthquake network is studied. Through the analysis of the real data taken from California and Japan, it is found that the values of the clustering coefficient exhibit a specific behavior around the moment of a main shock: the coefficient remains stationary before a main shock, suddenly jumps up at the main shock, and then slowly decreases to become stationary again. Thus, the network approach to seismicity dynamically characterizes main shocks in a peculiar manner.
\end{abstract}

Keywords: Earthquake network, Clustering coefficient, Dynamical evolution

\section{INTRODUCTION}

It is known in seismology [1] that an earthquake can be triggered by its foregoing one more than $1000 \mathrm{~km}$ away. This implies that event-event correlation in seismicity is enormously long-ranged, analogous to critical phenomena. This strong event-event correlation is responsible for specific statistical properties of seismicity. For example, the distributions of spatial distance [2] and time interval [3, 4] between two successive earthquakes radically differ from the Poissonian. These facts make it natural to put a working hypothesis that at the statistical level two successive events can be regarded to be indivisibly correlated, no matter how distant they are.

The complex network approach enables one to describe such correlation in an efficient way. The purpose of this article is to show how a new possibility can be opened for revealing yet unknown dynamics of seismicity with the help of the concept of earthquake network introduced and studied in a series of recent works [5-10]. In particular, we focus our attention on evolution of the clustering coefficient of earthquake network. We shall see that the value of the clustering coefficient stays stationary before a main shock, suddenly jumps up at the moment of the main shock, and then slowly decreases to become stationary again. To ascertain universality of this finding, we analyze several strong shocks occurred in different geographical regions; they are the Joshua Tree Earthquake (M6.1, on April 23, 1992), the Landers Earthquake (M7.3, on June 28, 1992), and the Hector Mine Earthquake (M7.1, on October 16, 1999) in California (database: http://www.data.scec.org), and the KushiroOki Earthquake (M7.1, on November 29, 2004) in Japan (database: http://www.hinet.bosai.go.jp). It is our opinion that the present result may offer a guiding principle for constructing/examining models of seismicity [11].

\section{CONSTRUCTION OF EARTHQUAKE NETWORK}

In this section, we succinctly explain the procedure of constructing an earthquake network originally proposed in Ref. [5].

We divide a geographical region under consideration into a lot of small cubic cells. We identify a cell with a vertex of a network if earthquakes (with any values of magnitude) occurred therein. Two successive events form an edge between two vertices. If they occur in the same cell, a tadpole (i.e., a self-loop) is attached to that vertex. These edges replace complex event-event correlation. Taking into account the fact that microscopic dynamics governing seismicity is still largely unknown, this approach is expected to offer an efficient representation of seismicity in view of science of complexity.

We make some comments on this construction. Firstly, it contains a single parameter, the cell size, which determines a scale of coarse graining. Once division by the cells is set, a seismic time series is unambiguously mapped to a growing stochastic network. Since any a priori rule is still not known for determining the cell size, it is important to examine the dependence of the property of an earthquake network on it, in general. (We shall not, however, discuss this point in the present work.) Secondly, an earthquake network is a directed graph. Directedness does not bring any difficulties to statistical analysis of connectivity (i.e., degree, the number of edges attached to the vertex under consideration) since in-degree and out-degree are identical for each vertex except the first and last ones in the analysis (i.e., in-degree and out-degree do not have to be distinguished one from another in the analysis of connectivity). However, directedness should be taken into account when the path length (i.e., the number of edges between a pair of connected vertices) and the period (implying after how many subsequent earthquakes the event returns to the initial vertex) are considered, for example. Finally, directedness has to be ignored and the path length should be defined as the smallest value among the possible numbers of edges connecting a pair of vertices, when small-worldness of an earthquake network is considered. There, tadpoles have to be removed and each multiple edge be replaced by a single edge. That is, a full directed earthquake network is reduced to a corresponding simple undirected graph (see Fig. 1).

An earthquake network and its reduced simple graph constructed in this way are highly complex: they are scale-free [5], of the small world [6], and locally treelike [7], exhibit hierarchical organization and assortative mixing [8], and possess the power-law period distribution [9]. A central reason why an earthquake network is heterogeneous is due to an empirical fact that aftershocks associated with a main shock tend to return to the locus of the main shock, geographically, and therefore the vertices of main shocks play roles of hubs of the 

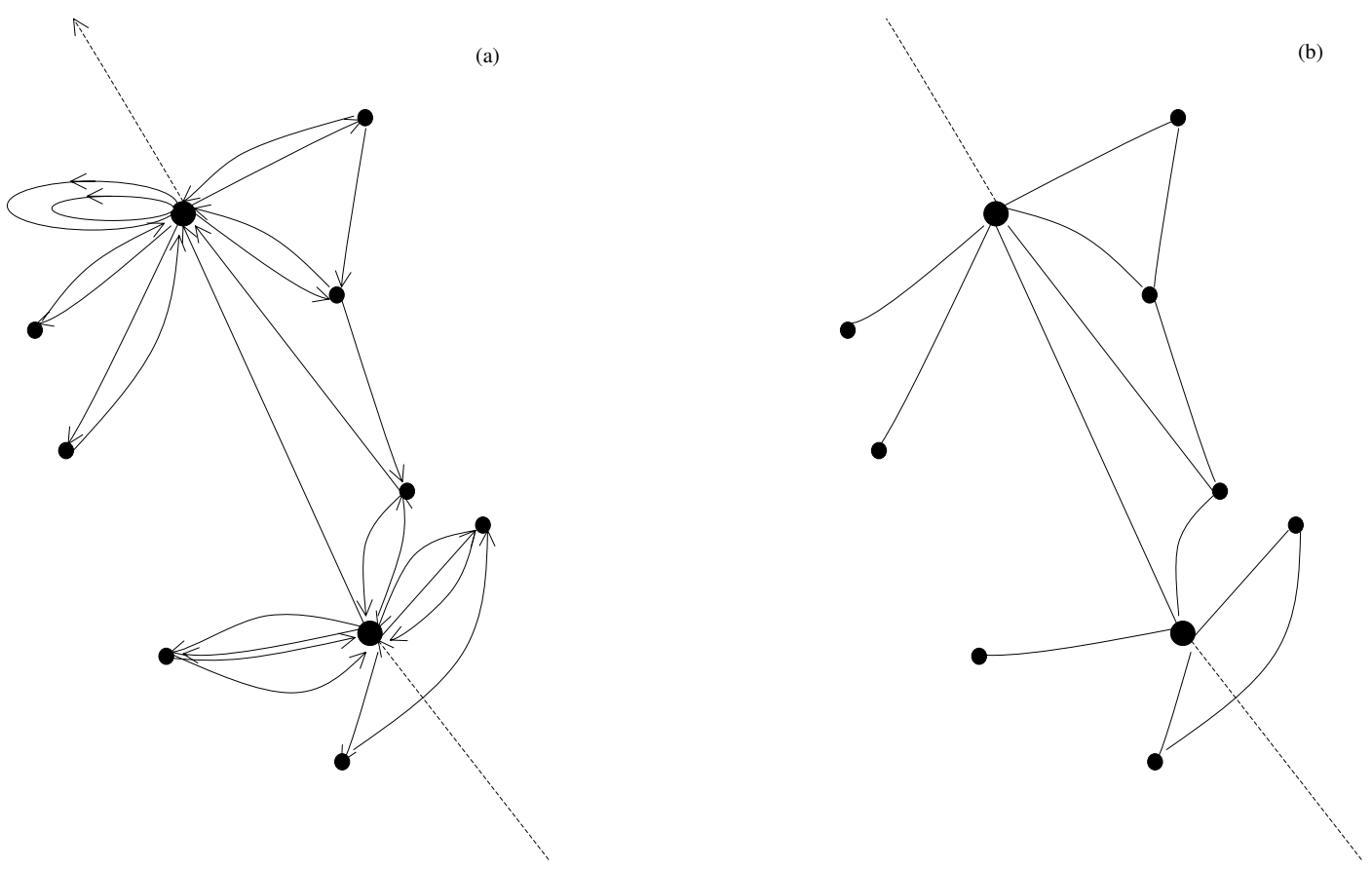

FIG. 1: Schematic description of earthquake network. (a) A full directed network, and (b) the simple undirected graph reduced from the full network in (a).

network.

\section{DYNAMICAL EVOLUTION OF THE CLUSTERING COEFFICIENT}

There are several important quantities that characterize the topological and statistical structures of complex networks. It is of obvious interest to see if they can play some roles in revealing salient features of seismicity, especially main shocks. Here, we focus our attention on the clustering coefficient [12] and pursue dynamical evolution of its value. Our main concern is about its behavior around the moment of a main shock [10].

The clustering coefficient is defined for a simple undirected graph with $N$ vertices as follows. Let $A=\left(a_{i j}\right)(i, j=$ $1,2, \ldots, N)$ be an adjacency matrix. $a_{i i}=0$ and $a_{i j}=1(0)$ if the $i$ th and $j$ th vertices are connected (unconnected) by an edge. Then, the clustering coefficient, $C$, is given by

$$
C=\frac{1}{N} \sum_{i=1}^{N} c_{i}
$$

where

$$
\begin{gathered}
c_{i}=\frac{e_{i}}{e_{i}^{\max }}, \\
e_{i}=\left(A^{3}\right)_{i i},
\end{gathered}
$$

$$
e_{i}^{\max }=\frac{k_{i}\left(k_{i}-1\right)}{2}
$$

and $k_{i}=\sum_{j=1}^{N} a_{i j}$ the value of connectivity of the ith vertex. As clear from Eq. (3), $c_{i}$ quantifies the tendency that a triangle loop is attached to the $i$ th vertex. Eq. (4) is nothing but the maximum value of $e_{i}$, which is realized when $k_{i}$ neighboring vertices are fully connected.

For each of the Joshua Tree Earthquake, the Landers Earthquake, the Hector Mine Earthquake, and the Kushiro-Oki Earthquake, we take an interval of the seismic time series containing the event, divide it into many segments, and construct a reduced simple earthquake network from each segment that is fixed to be 240 hours long. The network evolves in time over the collection of the segments. Regarding divisions of the geographical regions, the cell size is taken to be a typical one, $10 \mathrm{~km} \times 10 \mathrm{~km} \times 10 \mathrm{~km}$.

The result is presented in Fig. 2. There, one can appreciate a remarkable universal behavior: the clustering coefficient stays stationary before a main shock, suddenly jumps up at the moment of the main shock, and then decays slowly. It was suggested in Ref. [10] (in which only the data from California was analyzed) that the slow decay actually follows a power law. Thus, the present complex-network approach to seismicity can characterize main shocks and aftershocks in a peculiar manner. 

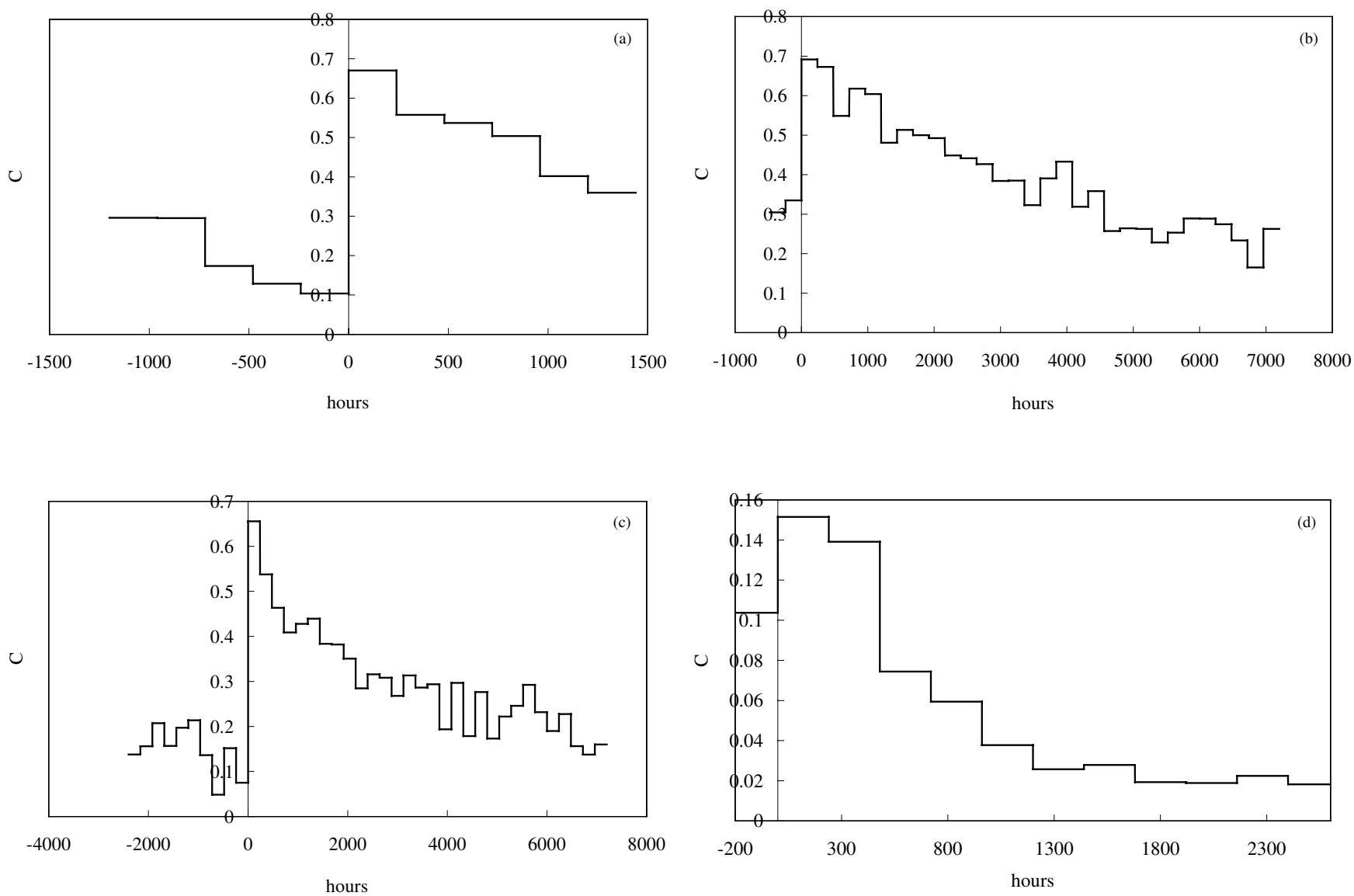

FIG. 2: Evolution of the (dimensionless) clustering coefficient during each 240 hours. (a) The Joshua Tree Earthquake, (b) the Landers Earthquake, (c) the Hector Mine Earthquake, and (d) the Kushiro-Oki Earthquake. The moments of the main shocks are located at time zero.

\section{CONCLUDING REMARKS}

We have shown how the present network approach to seismicity characterizes main shocks in a peculiar manner. In particular, we have seen that the clustering coefficient of an evolving earthquake network remains stationary before a main shock, suddenly jumps up at the moment of the main shock, and then slowly decays to become stationary again. We notice that, although the present discovery might remind one of the Omori law [13], they are not related to each other directly. This is because, in the definition of the clustering coefficient, tadpoles are removed and each multiple edge is replaced by a single edge: that is, a number of events are eliminated in the present analysis.

\section{Acknowledgments}

This article is based on the invited talk given by S. A. at the International Conference on Nonextensive Statistical Mechanics-Foundations and Applications (October 27-31, 2008, Foz do Iguaçu, Paraná, Brazil). S. A. thanks the organizers of the conference for the hospitality extended to him. His work was also supported by a Grant-in-Aid for Scientific Research from the Japan Society for the Promotion of Science.
[1] D. W. Steeples and D. D. Steeples, Bull. Seismol. Soc. Am. 86, 921 (1996).

[2] S. Abe and N. Suzuki, J. Geophys. Res. 108 (B2), 2113 (2003).

[3] Á. Corral, Phys. Rev. Lett. 92, 108501 (2004).

[4] S. Abe and N. Suzuki, Physica A 350, 588 (2005).

[5] S. Abe and N. Suzuki, Europhys. Lett. 65, 581 (2004); Nonlin. Processes Geophys. 13, 145 (2006).

[6] S. Abe and N. Suzuki, Physica A 337, 357 (2004).

[7] S. Abe and N. Suzuki, Physica A 388, 2511 (2009).

[8] S. Abe and N. Suzuki, Phys. Rev. E 74, 026113 (2006).
[9] S. Abe and N. Suzuki, Eur. Phys. J. B 44, 115 (2005).

[10] S. Abe and N. Suzuki, Eur. Phys. J. B 59, 93 (2007).

[11] T. P. Peixoto and C. P. C. Prado, Phys. Rev. E 74, 016126 (2006).

[12] D. J. Watts and S. H. Strogatz, Nature (London) 393, 440 (1998).

[13] F. Omori, J. Coll. Sci. Imper. Univ. Tokyo 7, 111 (1894). T. Utsu, Geophys. Mag. 30, 521 (1961). 